\title{
Interpretation of Orofacial Pain Symptoms in the Basis of New Findings in the field of Central and Peripheral Sensitizations
}

\author{
Yang $\mathrm{Gu}^{*}$ \\ Department of Oral and Maxillofacial Sciences, Dalhousie University, Canada
}

Received: March 19, 2018; Published: April 03, 2018

*Corresponding author: Yang Gu, Oral Pathologist, and Director of Oral Pathology Clinic, Department of Oral and Maxillofacial Sciences, Faculty of Dentistry, Dalhousie University, 5123 room, 5981 University Avenue, Halifax, NS, Canada, Tel: 9024941409; Fax: 902-494-6411; Email: yanggu@dal.ca

\begin{abstract}
Aims: The paper provided an effective way of analyzing orofacial pain cases based on new findings in the field of central and peripheral sensitizations.

Methods: The non-systematic review was undertaken by searching English literature in the PubMed by pertinent keywords. Case reports and publications with weak levels of evidence were excluded.

Results: Central and peripheral sensitizations affect the pain perception mainly in the way of of an imbalance of neurotransmitters, an oversensitivity of neurotransmitter receptors, an initiation of neurogenic inflammation, and a combination of Vitamin $\mathrm{B}_{6}$ or $\mathrm{B}_{12}$ deficiency.

Discussion: Understanding the mechanism will be helpful for clinicians to interpret orofacial pain symptoms at the molecular level. A comprehensive chart was provided.

Keywords: Neurotransmitter; Neurotransmitter Receptor; Neuron; Central Sensitization; Peripheral Sensitization; Orofacial Pain

Abbreviations: SNC: Sensory Nucleus Complex; WDR: Wide Dynamic Range; VPM: Ventral Postero-Medial; CGRP: Calcitonin Gene-Related Protein; GPCRs: G-Protein Coupling Receptors; VGICs: Voltage-Gated Ion Channels; GABA: Gamma-Aminobutyric Acid; RF: Reticular Formation; NE: Nor-Adrenaline; BD: Bradykinin; CCK: Neuropeptide Chole-Cysto-kinin; NO: Nitric Oxide
\end{abstract}

\section{Introduction}

The conception of peripheral and central sensitizations regarding a pain perception was proposed by Schmidt RF in 1991 [1] on the strength of the search result in the PubMed. The article summarized new findings in the field and interpreted orofacial pain symptoms at the molecular level. It will be helpful for clinicians to identify etiologies and set-up treatment plans.

\section{Method}

The non-systematic review identified recent original research papers, systematic reviews, meta-analysis articles and narrative reviews from author input supplemented by the PubMed search of terms including neurotransmitter, a neurotransmitter receptor, neuron, peripheral sensitization, central sensitization and orofacial pain. An academic book was cited too. Case reports and publications with weak levels of evidence were excluded.

\section{Results}

\section{Pain Perception and Pain Inhibitory Pathway}

In a normal situation, nociceptive signals are deducted by the pain inhibitory pathway before the pain perception is confirmed in the sensory cortex. The intensity of pain that we can feel is lower than that the initial nociceptive signal has.

The Afferent of Nociceptive Signals [2]: Trigeminal ganglion is the site of the first neuron for processing initial nociceptive signals. Peripheral nerve endings extend to the orofacial region, while the central ending reaches the trigeminal sensory nucleus complex (SNC) in the brain stem that is subnucleus caudalis, oralis and interpolaris. The second nociceptive neurons are within the wide dynamic range (WDR) adjacent to the SNC, while the third nociceptive neurons seat at the ventral posteromedial region (VPM) within the thalamus. Axons of Nociceptive neurons process the signal transduction by the transmembrane influx and efflux of cations including sodium, chloride, potassium, and calcium. How does an axon communicate with the following neuron? The intracellular calcium helps vesicles containing neurotransmitters infuse with the pre-synaptic membrane and then release neurotransmitters into the synapse, which binds to specific receptors in the post-synaptic membrane. Nociceptive signals eventually reach the sensory cortex after passing through three neurons and two synapses. 
Nociceptive Neurotransmitters and Receptors: They are substance $\mathrm{P}$ binding the neurokinin 1 receptor (NK1 receptor) [3], calcitonin gene-related protein (CGRP) binding the CGRP receptor [4] and Glutamate binding the Glutamate receptor [5]. Those are released from pre-synaptic terminals and activate 2 nd and 3rd neurons and eventually reach the sensory cortex. NK-1 receptor and CGRP receptor belong to G-protein coupling receptors (GPCRs) group. Glutamate receptors including NMDA receptor and AMPA receptor belong to Voltage-gated ion channels (VGICs) group.

Inhibitory Neurotransmitters and Receptors: Two synaptic spaces at WDR and VPM are locations where inhibitory neurotransmitters work on [6]. Gamma-aminobutyric acid (GABA) [7] and Glycine [8] produced by the reticular formation (RF) interneurons in WDR inhibit the second neurons to accept Nociceptive signals. Another four neurotransmitters inhibit 2 nd and 3rd neurons to transmit nociceptive signals. They are Serotonin (5-HT) [9] produced by the nucleus raphe, noradrenaline (NE) [10] produced by the locus coeruleu in the limbic system, Endogenous opioids [2] and cannabinoids [11] produced by hypothalamus and pituitary gland. 5-HT1A/D receptors binding 5-HT, alpha2 receptors binding NE, Opioid receptors binding endogenous opioids and Cannabinoid receptors binding cannabinoids all belong to the GPCR alpha 1 group. Opioid receptors at pre-synaptic terminals and post-synaptic membranes are distributed at the cortex, thalamus, periaqueductal gray, spinal cord and peripheral nerve endings [2].

\section{Peripheral Sensitization}

Peripheral sensitization is increased responsiveness and a reduced threshold of nociceptive neurons in the periphery to the stimulation of their receptive fields. (Definition from International Association of Study Pain www.iasp-pain.org/taxonomy)

Increased Responsiveness: Continuous tissue damage maintains a continuous high concentration of noxious stimuli. Noxious stimuli include prostaglandins (PGs), Bradykinin (BK), neurotrophins and cations [2]. Damaged cells release PGs to recruit immune cells. At the same time, PGs bind GPCR alpha s. Irritated vessels trigger BK formation that cleaves from kininogens in plasma. BK links BK1/2 receptors. Broken nerves produce neurotrophins for the reinnervation. Simultaneously neurotrophins attach with transforming tyrosine kinase receptor A (Trk A receptor). GPCR alpha s, BK1/2 receptors, Trk A receptors and NK1 receptors are located at the peripheral endings of nociceptive nerves (C-fiber and A-beta fiber).

Damaged cells release cations counting sodium, potassium, and calcium who bind Voltage-gated ion channels (VGICs) of the terminal ending of C-fibre and A-beta fiber. The peripheral sensitization subsides when PGs, BK, neurotrophins, and cations are diluted; otherwise, patients have a continuous pain. In addition, Mast cells release Substance $P$ that binds NK1 receptors [12]. Substance P and Bradykinin at the peripheral region decrease the threshold of the temperature afferent fiber [2]. Therefore, patients have a burning sensation. On other hands, the Vitamin B12 deficiency increases the risk of demyelination. It may increase the responsiveness to nociceptive signals [13].
Reduced Threshold: A-beta fiber has a lower threshold of depolarization than C-fibre, while C-fibre relatively is very easy to be damaged [14]. In condition of a persistent and severe tissue injury, A-beta fiber replaces C-fibre to transmit nociceptive signals [15]. The lower threshold in A-beta fiber renders nociceptive signals set off action potentials easily. A-beta is a mechanoreceptive fiber too. Accordingly, patients present with allodynia, a light touch triggering pain. When A-beta fiber begins to transmit nociceptive inputs the peripheral inhibitory mechanism will stop to work because A-beta fiber doesn't have mu opioid receptors, which are only present in C fibre [2]. On that account, a chronic pain doesn't respond to opioid drugs.

\section{Neurogenic Inflammation:}

Continuously activated 1st neurons produce neuropeptides peripherally as an axon reflex that is substance P, neurokinin, calcitonin gene-related protein (CGRP) and Glutamate [2,3]. The involving field of neuropeptides is beyond the tissue injury area because of the distribution of the nerve branches. Therefore, patients have a radiating pain. Neuropeptides have an independent function of vasodilation and permeability. In addition, substance P stimulates mast cell degranulation by binding substance $P$ receptors $[12,16]$. Granules and secretions from mast cells coordinate with the preceding inflammation. The consequence is the surrounding field adjacent to the tissue injury area has an inflammation too. It also magnifies the original inflammation. Clinically patients have hyperalgesia, an over-response to pain stimuli. Substance P also stimulates platelets producing 5-HT and mast cells producing histamine. The symptom will be burning sensation with a reddish and edematous appearance.

\section{Central Sensitization}

Increased responsiveness of nociceptive neurons in the central nervous system to their normal or subthreshold afferent input. (Definition from International Association of Study Pain www.iasppain.org/taxonomy)

Increased Responsiveness to the Normal Afferent Input: Reportedly anxiety $[17,18]$ or a higher level of dopamine $[19,20]$ accompanies with a lower level of GABA and Glycine. Neuropeptide cholecystokinin (CCK), an antagonist of endogenous opioids, will be produced in the situation of chronic pain [2]. The level of 5-HT and $\mathrm{NE}$ is lower in the situation of depression. The activated Histamine Receptor 3 is related to interneurons in the WDR spontaneously firing [21]. On other hands, Vitamin B6 is the cofactor of an enzyme of Glutamate decarboxylase, which is the way of the GABA synthesis. Deficiency of Vitamin B6 relatively reduces the amount of GABA [22].

Increased Responsiveness to the Sub threshold Afferent Input: In the scenario of hypoxia, ischemia or other CNS injury WDR Glia including microglia and astrocytes produces prostaglandins (PGs), nitric oxide (NO) and cytokines exciting $2^{\text {nd }}$ neurons by intracellular calcium accumulation that wipes off the magnesium blockage to NMDA receptor [2]. The consequence is $2^{\text {nd }}$ neurons expressing more NMDA receptors, which are more sensitive to Glutamate than AMPA receptors. 


\section{Discussion}

The orofacial pain includes toothache, myalgia, arthralgia, and neuralgia. Sometimes it is difficult to find the initial injury area because of complicated anatomy structures. It could be the best waybeyond the anatomy restriction to analyze orofacial pain symptoms at the molecular level (Table 1). The interpretation could be beneficial for clinicians to identify etiologies and set up an appropriate treatment plan.

Table 1: Symptoms,molecular mechanisms and underlying causes of the Orofacial Pain

\begin{tabular}{|c|c|c|}
\hline Orofacial pain symptoms & Potential mechanisms & Underlying causes \\
\hline Continuous pain & Persistent tissue injury or inflammation & \multirow{5}{*}{$\begin{array}{c}\text { Anxiety or depression } \\
\text { A higher level of dopamine } \\
\text { Active histamine receptor } 3 \\
\text { Vitamin B6 or B } 12 \text { deficiency } \\
\text { Headache caused by hypoxia }\end{array}$} \\
\hline Burning sensation & High level of substance P or bradykinin & \\
\hline Radiating pain & The first neuron is activated. & \\
\hline Allodynia & C-fibre is replaced by A-beta fibre. & \\
\hline Hyperalgesia & Mast cell degranulation & \\
\hline
\end{tabular}

\section{References}

1. Schmidt RF (1991) Neurophysiology of nociception and pain in deep tissue (skeletal muscle, tendon, joint, connective tissue). Schmerz 5(1): 13-28.

2. Miles T, Nauntofte B, Svensson P (2004) Clinical oral physiology, Quintessence Publishing Co Ltd, Copenhagen Chapter 4: 93-139.

3. Li WW, Guo TZ, Shi X, Sun Y, Wei T, et al. (2015) Substance P spinal signaling induces glial activation and nociceptive sensitization after fracture. Neuroscience 310: 73-90.

4. Melo Carrillo A, Noseda R, Nir RR, Schain AJ, Stratton J, et al. (2017) Selective Inhibition of Trigeminovascular Neurons by Fremanezumab: A Humanized Monoclonal Anti-CGRP Antibody. The Journal of Neuroscience 37(30): 7149-7163.

5. Vilar B, Busserolles J, Ling B, Laffray S, Ulmann L, et al. (2013) Alleviating Pain Hypersensitivity through Activation of Type 4 Metabotropic GlutamateReceptor. The Journal of Neuroscience 33(48): 18951-18965.

6. Drake RA, Hulse RP, Lumb BM, Donaldson LF (2014) The degree of acute descending control of spinal nociception in an area of primary hyper algesia is dependent on the peripheral domain of afferent input. J Physiol 592(16): 3611-3624.

7. Alavian F, Ghiasvand S, Sahraei H, Rafiei Rad M (2017) Intervention of the Gamma-Aminobutyric Acid Type B Receptors of the Amygdala Central Nucleus on the Sensitivity of the Morphine-Induced Conditionally Preferred Location in Wistar Female Rats. Addict Health 9(2): 110-117.

8. Armbruster A, Neumann E, Kötter V, Hermanns H, Werdehausen R, et al. (2018) The GlyT1 Inhibitor Bitopertin Ameliorates Allodynia and Hyperalgesia in Animal Models of Neuropathic and Inflammatory Pain. Frontiers in Molecular Neuroscience 10: 438.

9. Tian Z, Yamanaka M, Bernabucci M, Zhao MG, Zhuo M (2017) Characterization of serotonin-induced inhibition of excitatory synaptic transmission in the anterior cingulate cortex. Molecular Brain 10(1): 21.

10. Obata H (2017) Analgesic Mechanisms of Antidepressants for Neuropathic Pain. Int J Mol Sci 18(11): 2483.

11. Selley DE, Welch SP, Sim Selley LJ (2013) Sphingosine Lysolipids in the CNS: Endogenous Cannabinoid Antagonists or a Parallel Pain Modulatory System? Life Sci 93(5-6): 187-193.
12. Eller Smith OC, Nicol AL, Christianson JA (2018) Potential Mechanisms Underlying Centralized Pain and Emerging Therapeutic Interventions. Frontiers in Cellular Neuroscience 12: 35.

13. Shohei Yamamoto, Hideki Ono, Kazuhiko Kume, Masahiro Ohsawa (2016) Oxaliplatin treatment changes the function of sensory nerves in rats. Journal of Pharmacological Sciences 130(4): 189-193.

14. Van Den Broeke EN, Mouraux A, Groneberg AH, Pfau DB, Treede RD, et al. (2015) Characterizing pinprick-evoked brain potentials before and after experimentally induced secondary hyperalgesia. J Neurophysiol 114(5): 2672-268.

15. Paladini A, Fusco M, Coaccioli S, Skaper SD, Varrassi G (2015) Chronic Pain in the Elderly: The Case for New Therapeutic Strategies, Pain Physician 18(5): 863-876.

16. Liu M, Fitzgibbon M, Wang Y, Reilly J, Qian X, et al. (2018) Ulk4 regulates GABAergic signaling and anxiety-related behavior. Translational Psychiatry 8(1): 43.

17. Lueken U, Kuhn M, Yang Y, Straube B, Kircher, et al. (2017) Modulation of defensive reactivity by GLRB allelic variation: converging evidence from an intermediate phenotype approach, Translational Psychiatry 7(9): 1227.

18. Shimizu S, Sogabe S, Yanagisako R, Inada A, Yamanaka M, et al. (2017) Glycine-Binding Site Stimulants of NMDA Receptors Alleviate Extrapyramidal Motor Disorders by Activating the Nigrostriatal Dopaminergic Pathwa. Int J Mol Sci 18(7): 1416.

19. Alfredo Alaniz Palacios Ataulfo, Martínez Torr (2017) Antagonistic effect of dopamine structural analogues on human GABA 1 receptor. Scientific reports 7: 17385 .

20. Steve Mc Garaughty, Katharine L Chu, Marlon D Cowart, Jorge D Brioni (2012) Antagonism of Supraspinal Histamine H3 Receptors Modulates Spinal Neuronal Activity in Neuropathic Rats. The journal of pharmacology and experimental therapeutics 343 (1): 13-20.

21. Pena IA, Roussel Y, Daniel K, Mongeon K, Johnstone D, et al. (2017) Pyridoxine-Dependent Epilepsy in Zebrafish Caused by Aldh7a1 Deficiency. Genetics 207(4): 1501-1518.

22. Drake RA, Leith JL, Almahasneh F, Martindale J, Wilson AW, et al. (2016) Periaqueductal Grey EP3 Receptors Facilitate Spinal Nociception in Arthritic Secondary Hypersensitivity. The Journal of Neuroscience 36(35): $9026-9040$. 


\section{(C) (1) This work is licensed under Creative}

Submission Link: https://biomedres.us/submit-manuscript.php

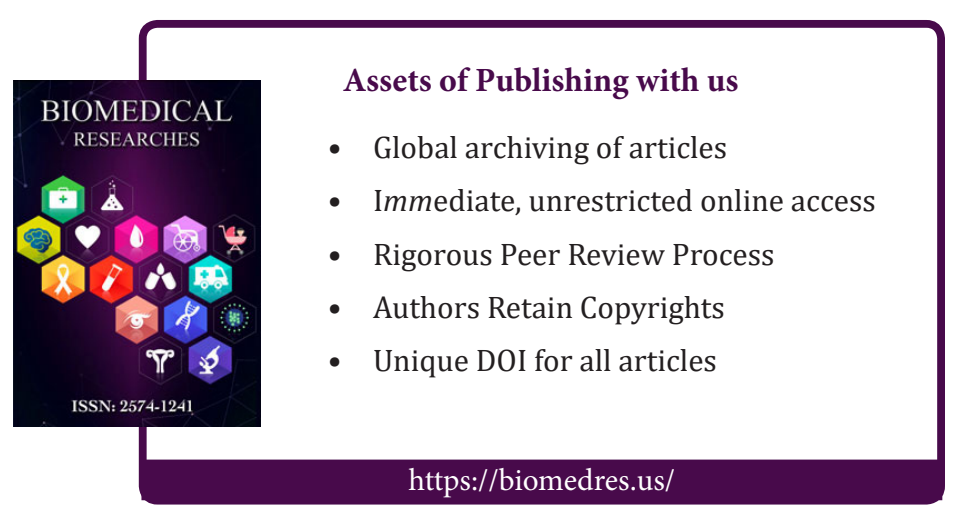

